An integrated strategy for large-scale investigation on protein core fucosylation stoichiometry based on Glycan-Simplification and Paired-Peaks-Extraction

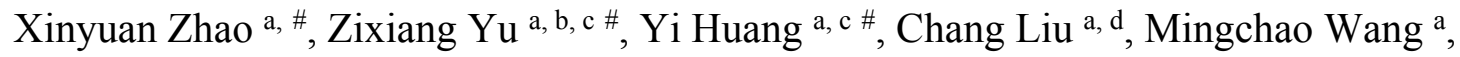
Xiaoyu Li ${ }^{\text {a, d }}$, Xiaohong Qian ${ }^{\text {a, Wantao Ying a * }}$

${ }^{a}$ National Center for Protein Sciences Beijing, State Key Laboratory of Proteomics, Beijing Proteome Research Center, Beijing Institute of Lifeomics, Beijing 102206, PR China

${ }^{b}$ Department of Pathology, Zhongshan Hospital, Fudan University, Shanghai 200032, P. R. China

${ }^{\mathrm{c}}$ Anhui Medical University, No. 81, Meishan Road, Hefei, Anhui, 230032, China

${ }^{\mathrm{d}}$ College of Life Science and Bioengineering, Beijing University of Technology, No. 100, Pingleyuan, Chaoyang District, Beijing 100124, China

\# These authors contribute equally to this work.

* Corresponding author email: yingwantao@mail.ncpsb.org 
Supplemental Table S1. The identified core fucosylation sites and their original stoichiometry values in human urinary samples.

Supplemental Table S2. The quantitation of core fucosylation sites after peptide identity propagation.

Supplemental Table S3. Detailed information of core fucosylation sites in HCC and healthy individuals. 


\section{Methods section:}

Materials and Reagents. Formic acid (FA), Trifluoroacetic acid (TFA), Ammonium bicarbonate, Iodoacetamide (IAA), Dithiothreitol (DTT), HPLC-grade acetonitrile (ACN), Endoglycosidase H, Endoglycosidase F3, and Protease inhibitor mixture were purchased from Sigma-Aldrich (St. Louis, MO., U.S.). Urea and Tris were purchased from USB (Ohio, USA). Sequencing-grade trypsin (trypsin) was purchased from Promega (Madison, WI, USA). Venusil HILIC $(5 \mu \mathrm{m})$ Silica Gel Filler was purchased from Tianjin Bonner Eager (Tianjin, China). 30KD ultrafiltration tubes and tubes were purchased from Millipore (Amicon Ultra, Ireland). Laboratory ultrapure water were prepared from Millipore A10 purification system (Billerica).

Urinary Protein Extraction. First, $10 \mathrm{~mL}$ of fresh urine sample was centrifuged at $12,000 \mathrm{x}$ g for $30 \mathrm{~min}$ to remove cell debris. Then, the urinary proteins were extracted by acetone precipitation. After that, lysis buffer ( $8 \mathrm{M}$ urea Tris- $\mathrm{HCl} 8.5,0.1 \mathrm{M}$ DTT) was used to redissolve the urinary proteins. The suspension was sonicated for $200 \mathrm{~W} \times$ $3 \mathrm{~s}($ working $) \times 3 \mathrm{~s}($ resting $) \times 10$ cycles and was next centrifuged at $16000 \mathrm{xg}$ for 5 min and the supernatant is collected for subsequent protein digestion.

Protein Digestion. The standard protein (RNase B, IgG) or the urinary proteins was dissolved in $8 \mathrm{M}$ urea, and transferred to a $30 \mathrm{KD}$ ultrafiltration tube. The tube was centrifuged at $14000 \mathrm{~g}$ for $20 \mathrm{~min}$. Proteins were reduced with $100 \mu \mathrm{l}$ of $20 \mathrm{mM}$ DTT for $4 \mathrm{~h}$ at $37^{\circ} \mathrm{C}$ and alkylated with $100 \mu \mathrm{l}$ of $50 \mathrm{mM}$ iodoacetamide for $30 \mathrm{~min}$ at $25^{\circ} \mathrm{C}$ in the dark. The buffer was subsequently replaced with $50 \mathrm{mM} \mathrm{NH}_{4} \mathrm{HCO}_{3}$ buffer $(\mathrm{pH}$ 7.8) by washing the ultrafiltration membrane three times. Sequencing-grade porcine trypsin (Promega, USA) was added to each sample with an enzyme to protein ratio of 1:50, and the solution was incubated overnight at $37{ }^{\circ} \mathrm{C}$. The peptides were collected via centrifugation at $14,000 \mathrm{~g}$ for $15 \mathrm{~min}$ and stored at $-80{ }^{\circ} \mathrm{C}$ after they were dried under vacuum.

HILIC Enrichment. Intact N-glycopeptides were enriched using HILIC enrichment method. Briefly, $100 \mu \mathrm{g}$ of tryptic peptides was desalted, dried and resuspended in $80 \%$ 
ACN/0.2\% TFA. Then, $5 \mathrm{mg}$ of Venusil HILIC (Agela Technologies) was washed three times for 10 min each with $0.1 \%$ TFA and $80 \%$ ACN/0.2\% TFA, followed by sample loading and rotating for $2 \mathrm{~h}$ at room temperature. Then, the mixture was transferred to a Pipet Tip (AXYGEN, USA) that was packed with C8 membrane and washed twice with $80 \% \mathrm{ACN} / 0.2 \%$ TFA. Glycopeptides bound to the HILIC column were eluted three times with $70 \mu \mathrm{l}$ of $0.1 \%$ TFA. The samples were dried by SpeedVac.

Endoglycosidase $\mathbf{H}$ and endoglycosidase F3 Digestion. The enriched glycopeptides were dissolved with $50 \mathrm{mM}$ ammonium acetate solution (pH5.5). Then, $2 \mu \mathrm{L}$ of Endoglycosidase $\mathrm{H}$ (Endo $\mathrm{H}$ ) was added. It was incubated in a $37^{\circ} \mathrm{C}$ incubator overnight and then dried by SpeedVac. After that, the sample was dissolved with 100 $\mathrm{mM}$ ammonium acetate solution (pH4.5). After $2 \mu \mathrm{L}$ of Endoglycosidase F3 (Endo F3) was added, the sample was incubated in a $37^{\circ} \mathrm{C}$ incubator overnight. The samples were desalted by $\mathrm{C} 18$ membrane and analyzed by LC/MS.

LC-MS/MS. Data acquisition was performed using the data-dependent mode in a Q-Exactive ${ }^{\mathrm{TM}}$ HF mass spectrometer (Thermo, USA) equipped with a nanoelectrospray ionization source and an Easy-nLC 1200 liquid chromatography system (Thermo, USA). The simplified glycopeptides were separated on a capillary column packed with $\mathrm{C} 18$ at a flow rate of $600 \mathrm{nl} / \mathrm{min}$. Mobile phase A (0.1\% FA) and mobile phase $\mathrm{B}(0.1 \% \mathrm{FA}$ in $\mathrm{ACN})$ were used, and the elution gradient used as follows: $5 \%$ mobile phase B; 0-14 min, 10\% mobile phase B; $14-51$ min, 22\% mobile phase B; 51 68 min, 36\% mobile phase B; 68-69 min, 100\% mobile phase B; 69-75 min, 100\% mobile phase B. The Q-Exactive HF was run in positive mode, and the $\mathrm{m} / \mathrm{z}$ range for the full-scan survey was from 300 to 2000 (AGC target was set $3 \mathrm{e}^{6}$ ), with a resolution of 120000 and a maximum ion injection time of $80 \mathrm{~ms}$. The top 20 most intensive precursor ions were selected for MS/MS analysis (AGC target was set $5 \mathrm{e}^{4}$ ), with a maximum ion injection time of $45 \mathrm{~ms}$. The NCE (normalized collision energy) value was set 27 .

Database searching and data processing. Raw files were first converted to MGF files by Proteome Discoverer 2.1(Thermo, USA) with default parameters and then 
treated by spectrum-picking and optimization tools written in Perl language. CF Nglycopeptide identification was processed with the method established in our laboratory ${ }^{8}$. For HN N-glycopeptide identification, the spectra with glycan diagnostic ions were extracted to exclude the interference of the nonglycopeptides. The candidates extracted above were then searched against the Homo sapiens Uniprot database (20205 entries) or the yeast Uniprot database Release_20170916(6721 entries) using the pFind 2.6 search engine. "N"s in the background database that match the N-glycosylation signature sequence $(\mathrm{NX}(\mathrm{S} / \mathrm{T} / \mathrm{C}), \mathrm{X}$ is not $\mathrm{P})$ were replaced with the letter "J", which was defined to have the same mass as Asn. Carbamidomethylation-Cys was set as a fixed modification. Variable modifications included oxidation-Met, acetylation of protein N-termini and a $203.079 \mathrm{Da}$ variable addition to $\mathrm{J}$ residues (_GlcNAc_J_203.079). A maximum of two missed cleavages were allowed. The tolerance of the precursor ion was $10 \mathrm{ppm}$, and the tolerance of fragment ions was 15 $\mathrm{mmu}$. The false-discovery rate at the peptide level was set to $<0.01$. Quantitative data processing consisted of first, the raw file was converted to mzXML format using Protein Wizard software; then, the readmzXML package was used to extract complete MS1 information from the mzXML file and generate a file in the format of a text file (including the actual retention time value (RT), scan number and MS1 peak intensity). In addition, the scan number and $\mathrm{m} / \mathrm{z}$ information corresponding to the simplified glycopeptides obtained from the pFind search were extracted. Using this information, combined with the detailed information in the text file, the RT and theoretical mass corresponding to the reduced glycopeptide were further obtained. Finally, the peak area of MS1 was extracted by the presumed RT value and mass as quantitative information of the simplified glycopeptides. If only the CF glycopeptide was identified, the corresponding putative $\mathrm{HN}$ glycopeptide was also extracted based on the accurate mass and retention time (assuming that $\mathrm{CF}$ was earlier than $\mathrm{HN}$ and within $1 \mathrm{~min}$ ). Using the above method, we obtained quantitative information on the two SGFs. The site-specific core fucosylation stoichiometry was calculated by the following formula: core fucosylation stoichiometry $(\%)=$ area $($ pep-CF $) /(\operatorname{area}($ pep-CF $)+$ area $($ pep-HN $))$. In 
PCA analyses, we defined the missing value as -1 (Figure 4) or 0 (Figure S4) so that it could be further numerically operated.

Heatmap were drawn by R package pheatmap. PCA method were implemented in R software.

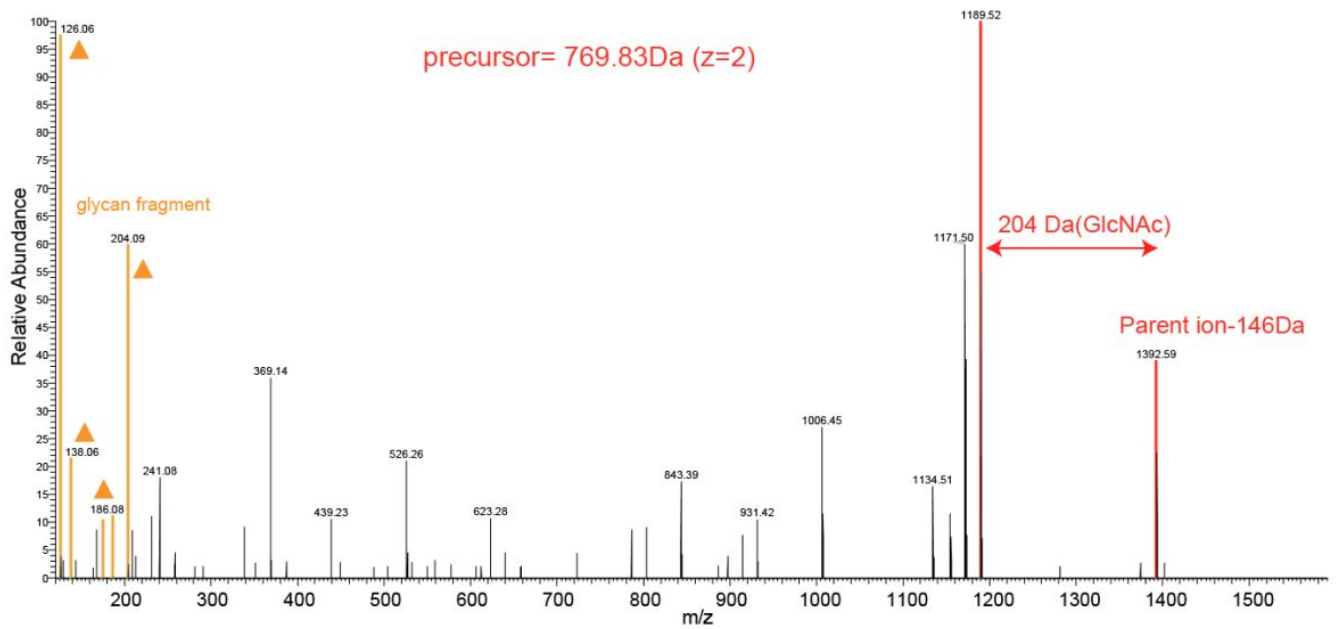

Figure S1. One representative MS/MS spectrum of CF N-glycopeptide. The parent ion $(\mathrm{z}=2)$ is $769.83 \mathrm{Da}$ and the parent ion with neutral loss of fucose $(146 \mathrm{Da}, 73 \mathrm{Da}$ for doubly-charged ions) is $1392.59 \mathrm{Da}$. Fragment peaks shown in yellow triangles are glycan diagnostic ions. 
A

\begin{tabular}{c|c|c|}
\hline Test & CF N-glycopeptides & HN N-glycopeptides \\
\hline 1 & 1 & 190 \\
\hline 2 & 0 & 191 \\
\hline 3 & 1 & 200 \\
\hline
\end{tabular}

B

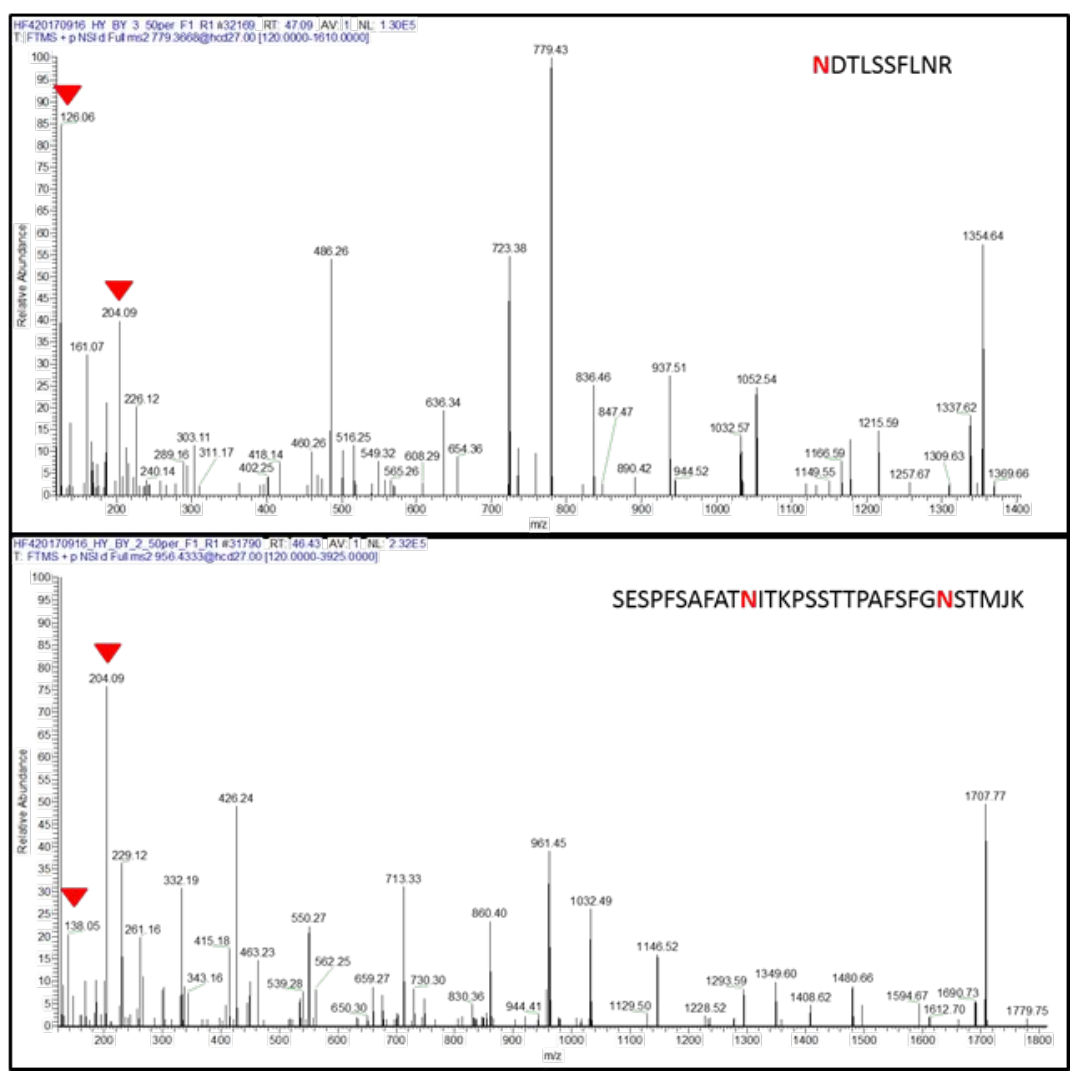

Figure S2. (A) List of pep-HN and pep-CF identification results from three tests of yeast proteins. (B) MS/MS spectra of the two false identification of CF N-glycopeptides in yeast proteins. 


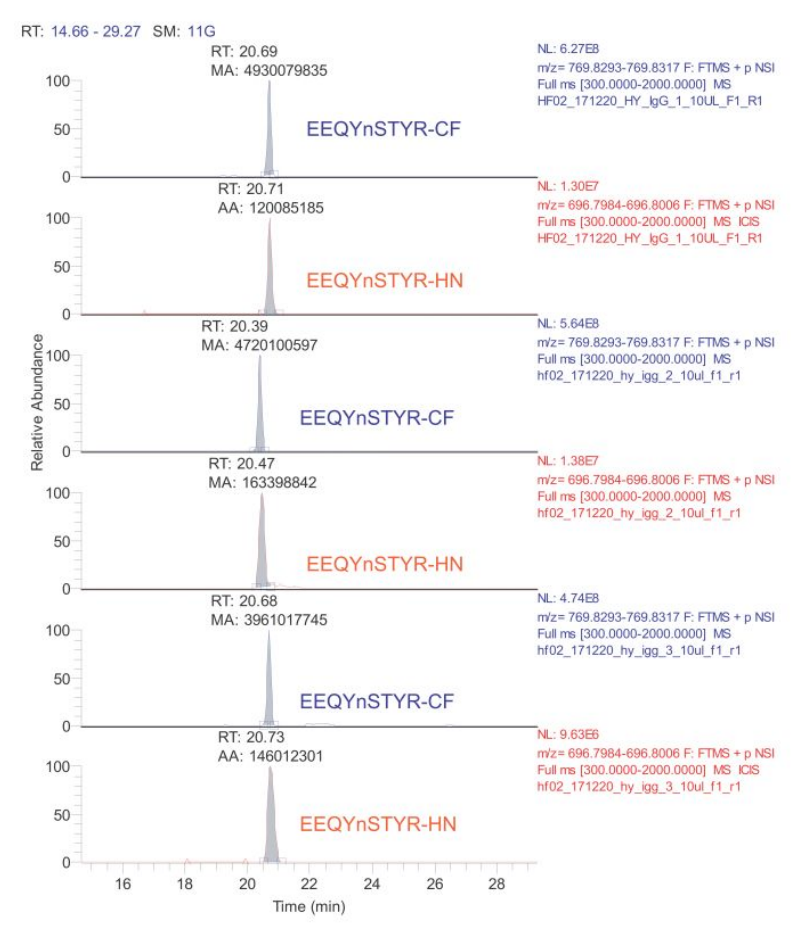

Figure S3. Extracted peaks of pep-CF and pep-HN in three biological tests.

Table S1. Peak area of pep-CF and pep-HN of IgG in three tests.

\begin{tabular}{|l|l|l|l|l|}
\hline test & Pep-CF area & Pep-HN area & total area & CF stoichiometry \\
\hline test 1 & 4930079835 & 120085185 & 5050165020 & 0.976221532 \\
\hline test 2 & 4720100597 & 163398842 & 4883499439 & 0.966540624 \\
\hline test 3 & 3961017745 & 146012301 & 4107030046 & 0.964448202 \\
\hline
\end{tabular}

Table S2. Quantitation of intact glycopeptide of EEQYnSTYR from IgG.

\begin{tabular}{|c|c|}
\hline Glycans & $\begin{array}{c}\text { Peak areas from } \\
\text { MaxQuant }\end{array}$ \\
\hline HexNAc(4)Hex(4)Fuc(1) & 12004000000 \\
\hline $\operatorname{HexNAc}(4) \operatorname{Hex}(3) \operatorname{Fuc}(1)$ & 6952900000 \\
\hline $\operatorname{HexNAc}(4) \operatorname{Hex}(5) \operatorname{Fuc}(1)$ & 2648900000 \\
\hline $\operatorname{HexNAc}(5) \operatorname{Hex}(3) \operatorname{Fuc}(1)$ & 872150000 \\
\hline $\operatorname{HexNAc}(4) H e x(3)$ & 793650000 \\
\hline $\operatorname{HexNAc}(4) H e x(5)$ & 766170000 \\
\hline HexNAc(4)Hex(5)Fuc(1)NeuAc(1) & 559700000 \\
\hline $\operatorname{HexNAc}(5) \operatorname{Hex}(4) \mathrm{Fuc}(1)$ & 132120000 \\
\hline $\operatorname{HexNAc}(5) \operatorname{Hex}(5) \operatorname{Fuc}(1)$ & 90646000 \\
\hline $\operatorname{HexNAc}(5) H e x(4)$ & 80104000 \\
\hline $\operatorname{HexNAc}(5) \operatorname{Hex}(3)$ & 59355000 \\
\hline $\operatorname{HexNAc}(4) H e x(4)$ & 55018000 \\
\hline $\operatorname{HexNAc}(3) \operatorname{Hex}(3)$ & 18743000 \\
\hline
\end{tabular}




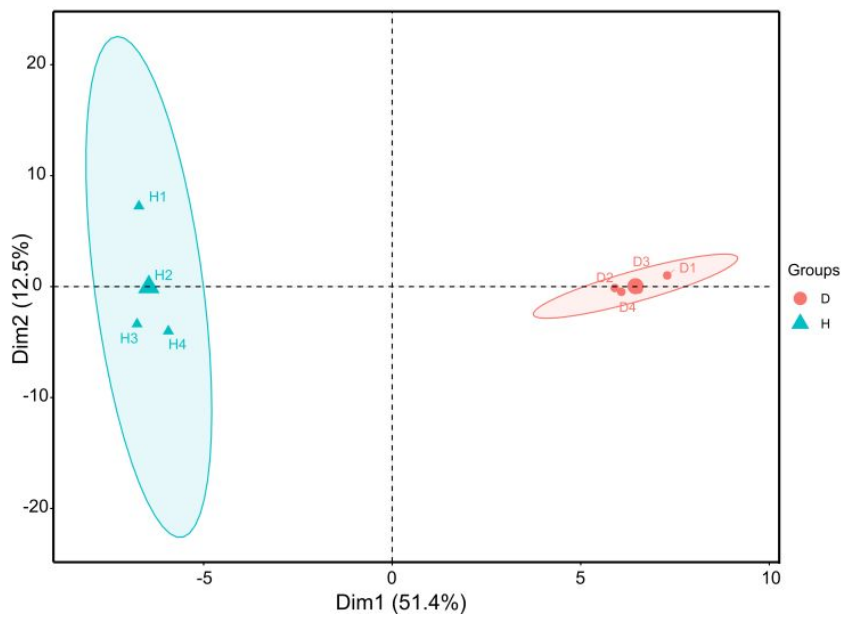

Figure S4 Principal component analysis of eight individuals based on their CF stoichiometries (missing values are replaced with value 0 ).

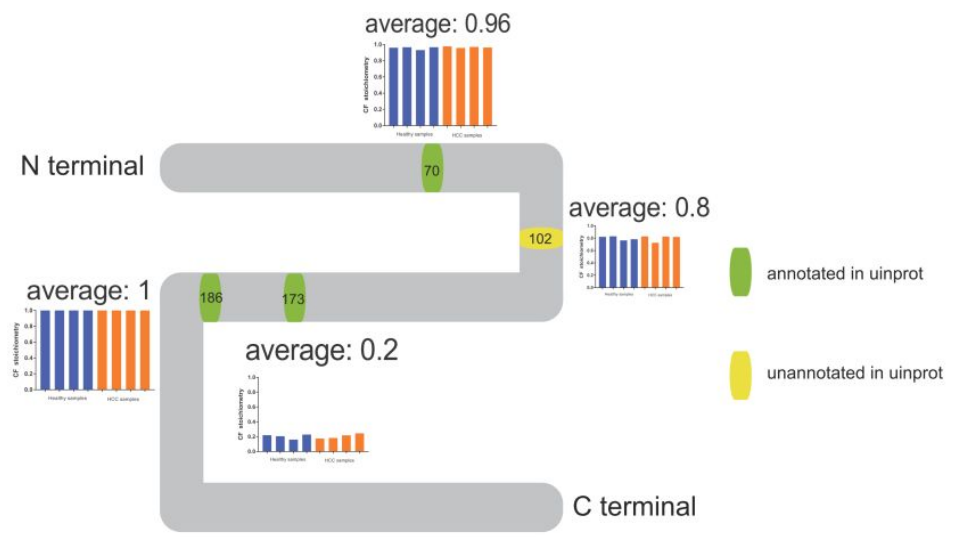

Figure S5. Distribution of CF stoichiometries in the protein ICOS ligand. 